\title{
Flexibilización y feminización de la mano de obra en la fase agraria de la citricultura de Uruguay
}

Flexibilization and feminization of the labor force in the agricultural phase of citriculture in Uruguay

\author{
Joaquin Cardeillac Gulla \\ Departamento de Sociología, Facultad de Ciencias Sociales, \\ Universidad de la República, Uruguay \\ joaquin.cardeillac@gmail.com \\ Julieta Krapovickas \\ Departamento de Sociología, Facultad de Ciencias Sociales, \\ Universidad de la República, Uruguay \\ krapovickasjulieta@gmail.com \\ Lorena Rodriguez Lezica \\ Departamento de Sociología, Facultad de Ciencias Sociales, \\ Universidad de la República, Uruguay \\ lorena.rodriguez.lezica@gmail.com \\ Alicia Isabel Migliaro González \\ Instituto de Psicología Social, Facultad de Psicología, \\ Universidad de la República, Uruguay \\ alicia.migliaro@gmail.com \\ Matias Carámbula Pareja \\ Departamento de Ciencias Sociales, Facultad de Agronomía, \\ Universidad de la República, Uruguay \\ mcarambula@fagro.edu.uy
}

\section{Resumen:}

En Uruguay, desde comienzos del siglo XXI, junto al crecimiento de los complejos agroindustriales de exportación asistimos a cambios en la organización del trabajo y en las formas de contratación del personal. Este trabajo analiza, mediante datos provenientes de estadísticas oficiales, el incremento de la mano de obra femenina en la fase agraria de la citricultura, haciendo hincapié en los procesos de jornalerización. Por otro lado, mediante el uso de herramientas cualitativas, da cuenta del sentido otorgado por actores empresariales al proceso de feminización de la mano de obra e incremento de la intermediación laboral. Palabras clave: Mercados de trabajo agrícolas, Demanda Laboral, Condiciones de Trabajo, Economía Feminista.

\section{Abstract:}

In Uruguay, since the beginning of the 21st century, together with the growth of agro-industrial export complexes, we have attended to changes in the organization of work and in the ways of hiring personnel. This paper analyses, using data from official statistics, the increase in the female labour force in the agricultural phase of citriculture, with emphasis on the processes of day labour. On the other hand, through the use of qualitative tools, it gives an account of the meaning given by business actors to the process of feminization of the labor force and increase of labor intermediation.

KEYWORDS: Agricultural Labor Markets, Labor Demand, Working Conditions, Feminist Economics. 


\section{INTRODUCCIÓN*}

En los últimos 20 años, buena parte de los estudios sociológicos y económicos acerca de lo rural y lo agropecuario en Uruguay han versado acerca del cambio o las transformaciones. Algunos se han centrado en el proceso de acaparamiento de tierras y extranjerización (Piñeiro, 2014 y 2010), mientras otros han discutido cambios en las formas de organizar la producción (Errea, Peyrou, Secco y Souto, 2011). En otros casos, el foco está en los procesos ocurridos en la estructura agraria (Cardeillac y Piñeiro, 2017; Riella y Romero, 2014), en el aumento de la importancia del trabajo asalariado (Cardeillac y Nathan, 2015) o en las implicancias de los cambios para el Uruguay rural y su futuro (Carámbula, 2015). Pero no todo ha sido cambio en el agro uruguayo. Investigaciones recientes sobre el trabajo asalariado rural han permitido constatar persistencias en procesos de privación de derechos y obstáculos al pleno goce de la ciudadanía (Juncal, Cardeillac, Moreira y Gallo, 2014; Cardeillac, Gallo y Juncal, 2015; Rodríguez Lezica, 2018).

En relación al trabajo asalariado rural, nuevas investigaciones han comenzado a paliar un vacío en torno al trabajo femenino asalariado (Rodríguez Lezica, 2014; Rodríguez Lezica y Carámbula, 2015; Cardeillac y Rodríguez Lezica, 2018). Esta problemática, que ha sido poco abordada en Uruguay, en otros países de la región tiene una larga tradición (Lara Flores, 1995; Salamea y Waters, 1995; Bendini y Pescio, 1996; Lastarria-Cornhiel, 2008; Soto Baquero y Klein, 2012; Mingo, 2015). De este modo, diferentes estudios han hecho visible cómo esta feminización del trabajo asalariado rural, en rubros no tradicionales de exportación, es producto de la nueva división internacional del trabajo que hace parte del reordenamiento del sistema agroalimentario mundial y la consecuente reconversión productiva de la agricultura latinoamericana (Lara Flores, 1995). Para Aranda (1988) la demanda específica de trabajo femenino en la exportación de productos frescos se debe principalmente a dos características atribuidas a las mujeres: la motricidad manual fina y los rasgos propios de una posición de subordinación, como ser la sumisión, dificultad de organización, carácter no conflictivo y aceptación de salarios bajos. A ello se debe agregar que los salarios más bajos que se pagan a las mujeres se fundamentan en la concepción patriarcal del salario femenino como una "ayuda" o complemento del salario masculino.

En Latinoamérica el crecimiento de los Complejos Agroindustriales de Exportación vinculados a Cadenas Globales de Valor (Gereffi, Korzeniewicz y Korzeniewicz, 1994) ha ido de la mano de cambios en la organización del trabajo, en las formas de contratación del personal, mostrando además una tendencia al incremento de la participación de las mujeres en el trabajo asalariado. Uruguay no es ajeno a estos procesos y se observan, desde comienzo del siglo XXI, incrementos en la participación de fuerza de trabajo femenina tanto en las fases agrarias como en las industriales de algunas importantes cadenas globales de valor agroalimentarias (Dominzain, 2003; Ipsen, 2010; Cardeillac y Rodríguez Lezica, 2018). Este artículo se focaliza en la producción citrícola, representativa de los complejos con una creciente participación de las mujeres en los procesos productivos. Si bien en la fase industrial -es decir en los empaques de fruta opackingsla participación de las asalariadas mujeres ha sido considerablemente mayor que en la fase agraria, es en la fase agraria de la cadena, en la cosecha de cítricos, donde se ha observado esta tendencia a su crecimiento.

Este trabajo tiene entonces como principales objetivos analizar: 1) el incremento de la mano de obra femenina en la fase agraria del rubro citrícola, 2) los procesos de jornalerización e incremento de la intermediación concurrentes con ese incremento y 3) el sentido otorgado por empresarios y contratistas a esos procesos.

\section{Cadenas Globales de Valor Agroalimentarias y desigualdades de género}

La noción de cadenas globales de valor (CGV) (Gereffi, Korzeniewicz y Korzeniewicz, 1994; Hopkins y Wallerstein, 1994; Sturgeon, 2008) resulta cada vez más ineludible al estudiar el trabajo asalariado rural o agropecuario en el Uruguay. Esto se debe a que, en los primeros años del siglo XXI, se concretó en el 
agro un avance mucho más intenso que en cualquier período histórico previo de formas capitalistas de producción asociadas a Sociedades con Contrato Legal, que dependen y se desarrollan para captar las ganancias derivadas del comercio internacional (Cardeillac, 2019). Las cadenas de valor de commodities suelen seguir tendencias contradictorias, a veces hacia la verticalización y concentración y, otras veces, hacia la dispersión y la competencia (Sturgeon, 2008; Cardeillac, 2013). En relación a los puntos que nos interesa discutir aquí, la teoría postula que se priorizará la integración vertical y la concentración toda vez que la posibilidad de acumulación de capital dependa de la disminución de costos de transacción, mientras que la tendencia a la diferenciación por vía de la subcontratación y la dispersión (procesos que coinciden con los observados en el período para el caso de la citricultura), sería el mecanismo adecuado para aumentar la acumulación abaratando el costo de la mano de obra (Hopkins y Wallerstein, 1994).

Por su parte, los estudios que abordan las cadenas globales de valor desde un enfoque de género indagan si el desarrollo de las CGV agrícolas ha constituido una oportunidad o una amenaza para el empleo femenino (FAO, FIDA y OIT, 2010; Bamber y Staritz, 2016). En el marco de mercados agrícolas cada vez más globalizados, en los cuales las cadenas de valor suelen estar controladas por corporaciones y supermercados nacionales o multinacionales, se observa que las oportunidades de trabajo de calidad para hombres como para mujeres son escasas, en tanto que los nuevos mercados de empleo se constituyen en:

...canales para transferir costos y riesgos a los eslabones más débiles de la cadena, particularmente las mujeres (...) perpetuar los estereotipos de género que mantienen a las mujeres en trabajos ocasionales y con menor retribución, y no necesariamente conducen a una mayor igualdad de género (FAO, FIDA y OIT, 2010, s/p).

Tanto en cadenas de valor tradicionales como modernas, mientras los varones predominan en los puestos permanentes, las mujeres acceden más a empleos temporales u ocasionales, inestables y flexibles, y cuando ocurre una desaceleración económica tienden a ser las primeras en perder su empleo (FAO, FIDA, OIT, 2010).

Así, si bien trabajadores y trabajadoras enfrentan desafíos importantes a raíz de la participación en las CGV, las desigualdades de género que estructuran la sociedad y la economía en general hacen que estos desafíos sean diferentes para unas y otros. En ese sentido, Bamber y Staritz (2016) muestran cómo las CGV se aprovechan de las desigualdades de género existentes, haciendo uso de mano de obra femenina para la elaboración de productos a bajo costo, y así la desigualdad de género se yergue como fuente de competitividad. De este modo, los empleos generados no desafían ni desmantelan la segregación ocupacional de género y los estereotipos relacionados con esta, sino que más bien se asientan sobre estos. Se evidencia, entonces, que la segregación horizontal disminuye en tanto las mujeres son empleadas en sectores tradicionalmente masculinizados. Su flexibilidad, su disposición, responsabilidad y compromiso con el trabajo son algunas de las características que se asignan a las mujeres, lo que las constituye en las favoritas para el empleo en industrias intensivas en mano de obra que operan bajo lógicas de gran competencia a nivel mundial (Bamber y Staritz, 2016). Otra dimensión de análisis tiene que ver con la sobrerepresentación de las mujeres en los trabajos estacionales e informales derivados de la necesidad de empleos con mayor flexibilidad y estacionalidad por presión de las empresas líderes en las CGV, ya que los modelos de empleo temporal o subcontratado se han convertido en centrales para mantener la competitividad (Bamber y Staritz, 2016).

En síntesis, los antecedentes nos permiten comprender la conveniencia que tiene para el capital el incremento de la mano de obra femenina en las CGV, en tanto las mujeres constituyen una mano de obra flexible, que hace parte del ejército laboral siempre disponible. Contrario al planteo de flexibilidad como necesidad desde las propias trabajadoras para poder lidiar con sus obligaciones domésticas y de cuidados, se trata de opciones que no son libres sino determinadas por restricciones culturales, económicas y de políticas públicas (Lara Flores, 1991) y producto de una división sexual del trabajo en la que las mujeres se especializan en el trabajo reproductivo (trabajo no remunerado, invisibilizado y desvalorizado) sobre el que se asienta la explotación capitalista (Federici, 2013). 
Nuestro enfoque postula, pues, la existencia de una complicidad estructural entre las desigualdades de género derivadas del sistema patriarcal que las especializa en el trabajo reproductivo, y las necesidades de acumulación del sistema capitalista, concretada en estrategias sinérgicas de feminización y “jornalerización”, orientadas a la reducción del costo de la mano de obra.

Es preciso en este punto definir por qué consideramos que el término jornalerización ofrece algunas ventajas descriptivas respecto a otros términos utilizados como zafralidad o temporalidad. Entendemos que existe una diferencia entre los distintos tipos de organización del trabajo y los distintos tipos de contratos laborales que es preciso puntualizar. En ese sentido, el trabajo zafral hace referencia a una cualidad particular de la tarea, describiendo una actividad laboral vinculada con procesos biológicos o estacionales de los productos, bienes o servicios que demanda un tipo específico y/o un incremento de la mano de obra en determinado período temporal acotado (Chiappe, Carámbula y Fernández, 2008). Por otra parte, la temporalidad refiere a un vínculo contractual laboral de duración acotada (Carámbula y Piñeiro, 2010). Temporalidad y zafralidad no necesariamente van de la mano ni deberían implicar necesariamente procesos de precarización laboral; sin embargo, existen íntimas relaciones fácilmente observables a nivel empírico. Los contratos temporales en tareas zafrales son una de las principales estrategias que las empresas utilizan para reducir los costos laborales (Tadeo, Palacios y Torres, 2005). Esta tendencia del capital, ampliamente abordada por autores de referencia (Neiman, 2010; Piñeiro, 2011; Hernández López, 2014) se engloba en el término jornalerización. Utilizamos este concepto para dar cuenta de la modalidad de organización del trabajo, que establece contratos temporales para la gestión de la mano de obra en tareas zafrales y cuya unidad de medida es la jornada laboral. Crecientemente, esta modalidad se acompaña de mecanismos de flexibilización y precarización.

El otro concepto que estructura teóricamente el artículo es el de la intermediación laboral. Para el análisis en este caso utilizamos el concepto de tercerización.

Quaranta y Fabio (2011) señalan que, en contextos de agriculturas reestructuradas, la intermediación laboral ha modificado su perfil, asumiendo una diversidad de modalidades y funciones, permitiendo así a las empresas utilizar estrategias muy flexibles de contratación y de registro de los trabajadores. Claramente, la distinción entre intermediación y tercerización forma parte de un vasto debate académico (ver por ejemplo Ermida Uriarte y Orsatti, 2009 o Basualdo y Esponda, 2014). Por las características particulares del mercado de trabajo del citrus, consideramos apropiado utilizar el concepto de la tercerización, asociando el mismo a los actores como el contratista, el cuadrillero y el enganchador. ${ }^{1}$

\section{BREVE DESCRIPCión DEL SECTOR CITRÍCOLA EN URUguAY}

La cadena agroexportadora del citrus en Uruguay tuvo un fuerte impulso en la década de los setenta, aprovechando el potencial del litoral norte del país para producir contra-estación las frutas que se consumen en los mercados del hemisferio Norte. Si bien la mayor parte de la producción se destina al mercado interno, Uruguay es un país exportador de cítricos. En 2018, de un total de 217 mil toneladas de cítricos producidos, la proporción exportada fue cercana al $40 \%, 38 \%$ se dedicó al mercado interno, $21 \%$ se destinó a la industria y el restante $2 \%$ corresponde a fruta que se perdió como descarte en el proceso de clasificación y empaque (Ackermann y Díaz, 2018).

Se estima que la fase agraria ${ }^{2}$ ocupa 14 mil hectáreas, con 6,9 millones de plantas totales, de las cuales el $83 \%$ se encuentra en producción (DIEA, 2018). Se caracteriza por ser intensiva en el uso de mano de obra, demandando aproximadamente 1,2 trabajadores por hectárea (unos 19.000 trabajadores en total) (Gabinete Productivo, 2010). El desarrollo de la cadena fue acompañado de un fuerte proceso de concentración, inversión, cambio técnico e integración vertical. En cuanto a la concentración, se estima que a fines de 2014 existían 452 productores citrícolas. No obstante, 6 empresas concentraban el $69 \%$ de las hectáreas bajo producción mientras que las otras 446 empresas acumulaban el $31 \%$ restantes (Gabinete Productivo, 2010). 
Los datos para 2018 dan cuenta de 421 productores citrícolas, siendo 5 empresas las que controlan $54 \%$ de las hectáreas y $57 \%$ de la producción nacional (DIEA, 2018).

De acuerdo a los últimos informes sectoriales (OPYPA, 2017 y 2018), la cadena se encuentra en niveles de producción y exportación por debajo de sus promedios históricos. En general, los informes coinciden en destacar que para recuperar los mercados perdidos o para acceder a nuevos mercados, es necesaria una reconversión varietal. Ruffier (2005) señala que los cítricos de Uruguay presentan pocas ventajas comparativas respecto de sus competidores, especialmente los productores de Sudáfrica. Allí los salarios son menores, el clima es mejor y los trayectos hacia Europa y a Oriente son más cortos (Ruffier, 2005). Además, Sudáfrica desde 2016 tiene un acuerdo de libre comercio con la Unión Europea, al igual que Chile y Perú (otros exportadores de fruta fresca) mientras que, a partir de 2014, los cítricos uruguayos no califican en el sistema general de preferencia (SGP) de la Unión Europea al haber subido la renta per cápita del país, lo que significó un cambio en el arancel, que subió 16 \% (Galván, 2013).

Un atenuante a la situación de pérdida de competitividad en el mercado europeo fue la apertura, en 2013, del mercado estadounidense. Esto implicó cambios varietales - avanzando hacia la producción de mandarinas sin semillas, por sobre la producción de naranjas-, además de cambios en materia sanitaria y en el packing. La importancia de este mercado se observa al analizar los datos provistos por el anuario de la OPYPA (2018) en el cual se señala que, en 2018, el 37 \% del valor de las exportaciones de cítricos se generó por ventas al mercado estadounidense. Esto representa un cambio, ya que Estados Unidos desplazó en 2018 por primera vez a la Unión Europea al segundo lugar de importancia (36 \% del valor exportado).

Para adecuarse al nuevo mercado estadounidense las empresas más grandes se encuentran realizando estas reconversiones varietales, lo cual genera una menor actividad en la cadena. Como resultado de esta coyuntura poco favorable para el sector, se resolvió un régimen especial de seguro de desempleo para los y las trabajadoras.

En síntesis, estamos frente a un sector en recesión respecto de su desempeño tradicional. Este aspecto es importante, ya que los antecedentes advierten que las CGV, en momentos así, priorizan abatir los costos de la mano de obra (Hopkins y Wallerstein, 1994; Cardeillac, 2013). En este sentido, el sector citrícola aparece como un escenario idóneo para estudiar los procesos concurrentes de feminización y jornalerización conceptualizados en los términos que aquí proponemos: en tanto mecanismos orientados a aliviar costos, mediante el uso de desigualdades derivadas de un sistema patriarcal generador de condiciones de vida y trabajo muy específicas y diferentes para las mujeres.

\section{Abordaje Metodológico}

Para alcanzar los objetivos propuestos, empleamos un abordaje de "métodos mixtos", generando distintos tipos de evidencia (Creswell, 2014). Al complementar así las metodologías pudimos acceder, por un lado, a una dimensión descriptiva, extensa y, por el otro, a una dimensión simbólica, de intercambio de significados (Bericat, 1998). Para el abordaje cuantitativo recurrimos al análisis de los Censos Generales Agropecuarios (CGA) de 1990, 2000 y 2011, y para el cualitativo a entrevistas en profundidad a empresarios y contratistas en el litoral noroeste del país (en los departamentos de Salto, Paysandú y Río Negro).

La selección de los CGA como fuente de datos se debe a que el tipo de fenómeno que nos interesa analizar (jornalerización), así como en nivel de desagregación requerido para llegar a la población objeto de estudio (asalariados y asalariadas de la fase agraria de la citricultura), no pueden ser abordados con ninguna otra fuente de datos secundaria. ${ }^{3}$

De hecho, incluso trabajando sobre los CGA, la desagregación que realizan en 1990 y 2000 no permite atender específicamente al sector citrícola. Recién en 2011 las explotaciones dedicadas a la citricultura aparecen aparte. Por este motivo, el análisis de tendencias se hace para la fruticultura y recién al final se profundizará en los resultados que se obtienen para la citricultura en 2011. 
También es importante aclarar que los CGA tienen como unidad de análisis a las explotaciones, por lo que el conteo de población a partir de los mismos siempre resulta menor que el realizado por los Censos de Población. En ese sentido, es una fuente con un sesgo productivo, por lo que las dimensiones que miden de la población y la mano de obra son limitadas. Ahora bien, aun a pesar de estas limitaciones, resulta ser la única fuente adecuada al problema que queremos abordar, además de tener una muy buena cobertura, ser confiable y haber mantenido su periodicidad.

En cuanto al abordaje cualitativo, las entrevistas se realizaron entre febrero de 2018 y agosto de 2019. La muestra se compone de cuatro contratistas de mano de obra para la cosecha de citrus y cuatro gerentes de recursos humanos de las cuatro principales empresas citrícolas de Uruguay. Las entrevistas fueron semidirigidas, focalizadas en el origen y evolución del contratismo como modo de acceso a la mano de obra agrícola en el rubro, en la feminización de la mano de obra y en la creciente “jornalerización”. Luego, el análisis se realizó con ayuda de un programa que facilita la codificación y el trabajo con familias de códigos y redes de códigos.

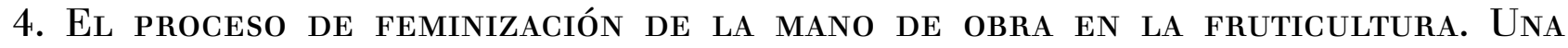
APROXIMACIÓN A TRAVÉS DE DATOS CENSALES

Para contextualizar los datos sobre fruticultura, resulta útil saber que la citricultura representaba en 2011 el $66 \%$ de los y las jornaleras dentro de la fruticultura y el $53 \%$ de los/as asalariados/as permanentes. Se observa así que el peso del sector citrícola en el trabajo asalariado de la fruticultura es elevado, y especialmente en el caso del trabajo asalariado jornalero.

Entre 1990 y 2011 se dio en la fruticultura una disminución del número de explotaciones y un aumento de la superficie promedio, mientras que la superficie total dedicada al rubro se mantuvo estable (Tabla 1).

TABLA 1

Fruticultura. Cambio en el número de explotaciones, en la superficie media y total y en el número de trabajadores/as, asalariados y asalariadas.

\begin{tabular}{|l|c|c|c|c|c|c|}
\hline & \multicolumn{3}{|c|}{ Número } & \multicolumn{3}{c|}{ Variación en base 1990 } \\
\hline & $\mathbf{1 9 9 0}$ & $\mathbf{2 0 0 0}$ & $\mathbf{2 0 1 1}$ & $\mathbf{1 9 9 0}$ & $\mathbf{2 0 0 0}$ & $\mathbf{2 0 1 1}$ \\
\hline Superficie Media & $\mathbf{4 5}$ & 50 & 64 & 100 & 111,7 & 143,4 \\
\hline Superficie Total & 65.870 & 61.919 & 64.386 & 100 & 94,0 & 97,7 \\
\hline Trabajadores/as & 6.198 & 6.020 & 7.332 & 100 & 97,1 & 118,3 \\
\hline Asalariados/as & 3.796 & 3.753 & 6.036 & 100 & 98,9 & 159,0 \\
\hline Asalariados & 3.492 & 3.400 & 4.570 & 100 & 97,4 & 130,9 \\
\hline Asalariadas & 304 & 353 & 1.465 & 100 & 116,2 & 482,0 \\
\hline Familiar Varón & 1.816 & 1.543 & 892 & 100 & 85,0 & 49,1 \\
\hline Familiar Mujer & 586 & 724 & 404 & 100 & 123,5 & 68,9 \\
\hline Explotaciones & 1.474 & 1.241 & 1.005 & 100 & 84,2 & 68,2 \\
\hline
\end{tabular}


En el caso de la mano de obra, en cambio, se observa una estabilidad entre 1990 y 2000 . Pero entre 2000 y 2011 hay un aumento que se explica por el incremento en la cantidad de asalariados/as y, particularmente, por el aumento del número de trabajadoras asalariadas.

Con estos procesos macro de trasfondo, la evolución del trabajo en el rubro es la que sigue:

1. El número total de trabajadores/as (familiares o asalariados/as) es en 2011 un 18,3 \% mayor que en 1990.

2. El número de trabajadores/as familiares varones y mujeres se reduce, siendo en 2011 el 49,1\% de los varones que se observaron en 1990 y $69 \%$ en el caso de las mujeres.

3. El número de asalariados/as aumenta. Los varones, $31 \%$, mientras que en el caso de las asalariadas el aumento es de $382 \%$, es decir un aumento 12 veces mayor que el de los varones (figura 1).

\section{FIGURA 1}

Fruticultura. Evolución en base 100 del número de trabajadores/ as familiares varones y mujeres y de asalariados y asalariadas

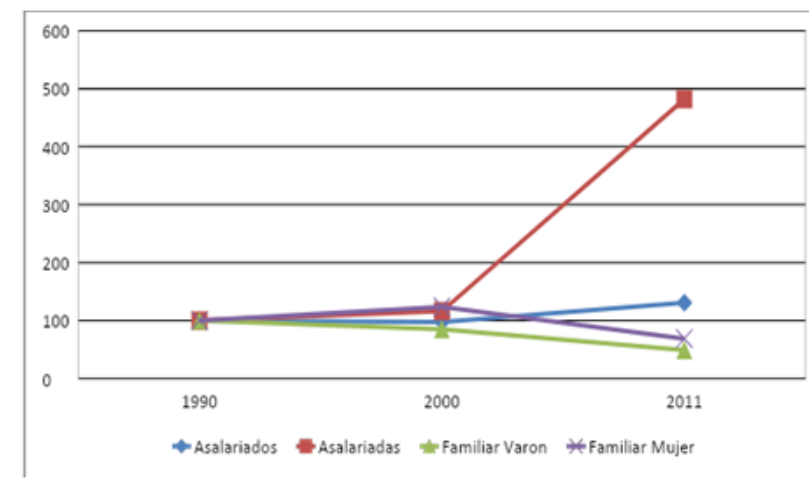

Fuente: CGA 1990, 2000 y 2011.

FIGURA 2

Fruticultura. Participación del trabajo familiar y asalariado por sexo

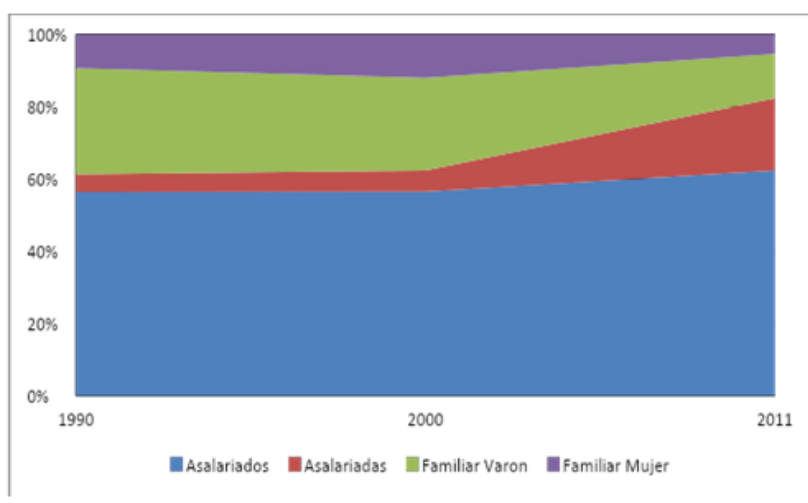

Fuente: CGA de 1990, 2000 y 2011.

En consecuencia, el proceso de asalarización del trabajo en la fruticultura se asoció a un proceso de feminización.

Un aspecto adicional, que implica una transformación relevante, es el cambio en la participación de asalariados/as permanentes y jornaleros/as. La figura 3 muestra cómo creció la mano de obra jornalera, llegando a ser en 2011 el tipo predominante en la fruticultura uruguaya. 
FIGURA 3

Fruticultura.Número de asalariados/as permanentes y jornaleros/as

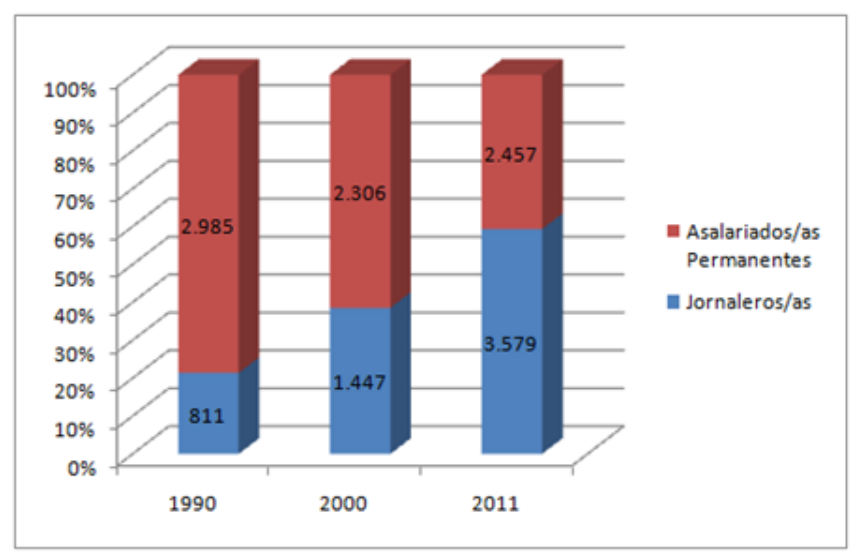

Fuente: CGA de 1990, 2000 y 2011.

En la citricultura, en términos del peso del trabajo familiar y asalariado hay un dominio claro del segundo, ya que el $84 \%$ del trabajo del rubro es realizado por asalariados y asalariadas. En ese sentido, la situación de la citricultura profundiza la tendencia que se observa en general para la fruticultura. A su vez, si analizamos la participación relativa del trabajo asalariado permanente y el jornalero resulta que casi $65 \%$ del trabajo asalariado del rubro es realizado por jornaleros y jornaleras (figura 4). Así, el sector funciona como un espacio en el que se expresa con claridad la importancia que ha adquirido la contratación temporal en el agro uruguayo.

FIGURA 4

Citricultura. Participación relativa sobre el trabajo asalariado total del trabajo permanente o temporal y según sexo. Año 2011.

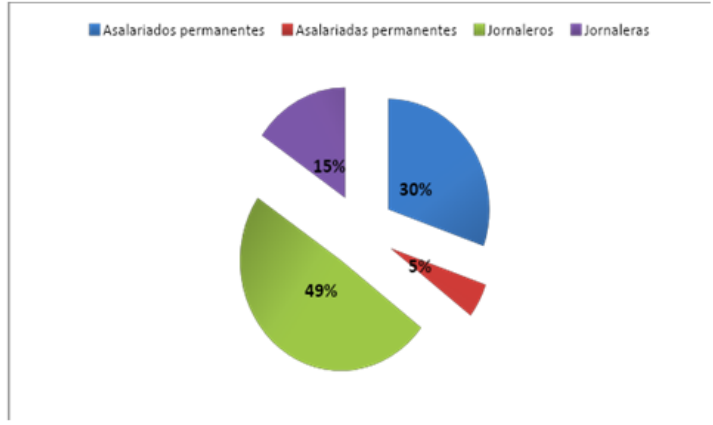

Fuente: CGA de 2011.

Los datos del CGA dan cuenta entonces que la participación de las mujeres en el trabajo dentro del rubro se asocia con un tipo de vínculo contractual: jornalera. De hecho, el peso de las mujeres asalariadas temporales de la citricultura triplica el peso de las asalariadas permanentes.

\section{LOS SENTIDOS OTORGADOS DESDE LOS ACTORES EMPRESARIALES LOCALES}

En la siguiente sección nos abocaremos al análisis del proceso de feminización del trabajo asalariado desde la perspectiva de algunos de los principales actores del proceso, es decir, los empresarios contratistas de mano de obra y los funcionarios de recursos humanos de algunas de las principales empresas citrícolas de Uruguay. Antes de ello, analizaremos la creciente participación de empresas contratistas en el sector, la cual entendemos es constitutiva del proceso de jornalerización, flexibilización y feminización de la mano de obra. 


\subsection{La tercerización de la mano de obra en la citricultura}

Las personas que trabajan en la cosecha del citrus en Salto, Paysandú y Río Negro forman parte de un sistema conformado por empresas madres (empresas citrícolas) y, en una tendencia creciente, encontramos ya sea la presencia de contratistas de mano de obra o cuadrilleros que organizan y gestionan y hasta tienen injerencia en la contratación del personal de zafra. Tanto en un caso como en el otro, la mano de obra se inserta de un modo muy laxo e informal en la estructura de la empresa, donde la rotación es muy alta.

La mano de obra del citrus en la región litoral del Uruguay es en general una mano de obra cautiva, con escasas oportunidades laborales (esta particularidad se agrava en localidades rurales como Villa Constitución, Belén, Pueblo Gallinal, más aisladas y sin la demanda de trabajadores/as para las actividades industriales o de servicios que se observa en las ciudades como Salto o Paysandú) (Cardeillac, Mascheroni y Vitelli, 2016).

Los contratistas, por otra parte, conforman empresas que son Sociedades de Responsabilidad Limitada. En general, operan a través de diferentes empresas; es decir, mientras trabajan con su propia empresa, conforman sociedades con otros contratistas en nuevas firmas empresariales. De este modo, encontramos diferentes empresas conformadas por los mismos empresarios contratistas. Los tercerizadores, a su vez, también venden servicios a empresas de otros rubros, ya sea reclutando mano de obra (para la cosecha de arándanos, por ejemplo) u otra clase de servicios al agro (maquinaria, servicios fitosanitarios, entre otros).

Del análisis de las entrevistas se desprende que, en los cuatro casos observados, su actividad como contratistas tercerizados empezó a pedido de la empresa madre, lo que da cuenta de un cambio en la política de organización del trabajo:

Lo de la tercerización surge porque ante la escasez de trabajo voy a la citrícola a ver si me pueden contratar los tractores... entonces "mire (...) lo que precisamos es gente que nos arrime cosecheros". "Y bueno: ¿cómo es el sistema?", me explicaron. Y'ta, armamos una SRL. Y al mes y medio ya estábamos trabajando. (Contratista 1, comunicación personal, abril de 2018).

Otro contratista, con más años en el rubro, señala el mismo origen: el pedido de la empresa. Este último, además, reconoce una evolución en el proceso de tercerización, que empezó con la aparición de empresas contratistas internacionales, hacia el año 2002. Actualmente, esas empresas no operan en el sector, sino que son ex trabajadores de la empresa madre, personal de recursos humanos o capataces, los que comenzaron a formar empresas tercerizadoras.

Yo trabajaba en la oficina de personal, de recursos humanos de [la empresa]. [La empresa] tuvo una etapa que empezó a reconvertir todo, (...) a tercerizar. Vino una internacional, Manpower, vino Addeco (...) Addeco habrá trabajado 4 o 5 años, se fueron, (...) y ahí surgió la invitación: -"si te animás vos", -"y si". (...) no es vocacional ni que lo pedí... se me fue dando. (Contratista 2, abril de 2018).

En la construcción de sentido de estos actores, el contratismo, su origen y la importancia de la tercerización, aparece asociado a la crisis del sector, a la necesidad de la empresa de "sacarse de encima el problema de la gente" (Contratista 3) y generar ahorro en gastos operativos y en logística, flexibilizando el manejo de una mano de obra jornalera y con alta rotación.

Yo creo que nosotros le aportamos a la estructura de trabajo dinamismo. (...) Por ej. hoy llueve. Es una llamada de teléfono que me hagan a mí y yo paro a 60 personas de salir al campo, ta? Me hago cargo yo. Y si hay que reorganizar ese grupo... y ta, surgió la posibilidad en tal lado no llovió, 15 personas, ¿̇te animas? Y yo entre los 60 míos busco 15, una combi y salgo. Una empresa grande como que se le hace pesado ese tipo de cosas. Nosotros aportamos dinamismo. (Contratista 2, abril de 2018).

Otro de los elementos que favorecen a la empresa madre es el costo de la mano de obra de gestión de recursos humanos: tercerizar es quitar un empleado de recursos humanos y convertirlo en contratista. La empresa gana en tanto se quita un empleado permanente y delega la contratación de empleados/as jornaleros/ as y el ex empleado gana en tanto al convertirse en contratista puede vender otros servicios (servicios de maquinaria, fumigación y/o contratación de trabajadores/as jornaleros/as para otros rubros) aunque no necesariamente gane más por la misma tarea.

Contratista 2: [Yo en mi empresa] Tengo un contador, la parte del BPS, por conocimiento, la hago yo, me ayuda un poco mi señora.

Entrevistadora: ¿ella forma parte de la empresa? 
C2: O sea, no. Los dos fuimos administrativos, conocemos más o menos del trabajo y sabe... (...) en eso es parte de la diferencia que hablábamos hoy, en vez de una oficina de recursos humanos con 10 personas, 15, lo hacemos nosotros. (Contratista 2, comunicación personal, abril de 2018).

Por otro lado, al mismo tiempo se afirma que los costos de la contratación de trabajadores/as vía empresas tercerizadas son más altos que contratar directamente en la empresa. "El costo del trabajo en sí no lo bajas, porque somos más caros. La empresa, en su sistema de aportes, hay creo hasta un $15 \%$ menos que paga si el empleado es propio" (Contratista 2, abril de 2018).

Este asunto es importante, ya que cuestiona la racionalidad económica de la tercerización. No obstante, tanto algunos empresarios como asalariados y asalariadas entrevistadas advierten sobre los elevados niveles de subdeclaración que caracterizan a la actividad de algunos contratistas.

Otro conjunto de entrevistas se realizó a representantes de las empresas. Al analizar estos discursos, se observa que los gerentes de recursos humanos de las empresas hacen una suerte de deslinde de responsabilidad sobre el accionar de las empresas contratistas. Así, por ejemplo, el responsable de recursos humanos de la empresa 1 -que no permitió grabar la entrevista- hizo hincapié en que sólo podía ofrecer datos de "los trabajadores de la casa" (directamente contratados) y que no tenía ningún tipo de control sobre los/as trabajadores/as dependientes de los contratistas. En relación a las contradicciones con los contratistas llama la atención que, en números globales, manejaban un número sensiblemente menor de asalariados/as. Mientras para el gerente de RRHH la zafra de esta empresa involucra 450 trabajadores, para los contratistas la misma llegaba a 900 personas, el doble. A su vez, frente a la pregunta de por qué la empresa decidió tercerizar la cosecha y qué beneficios obtiene de la misma, la respuesta fue que "la figura del contratista está más arriba de la gente" (Gerente RRHH empresa 1) y que se cosecha más volumen de fruta. "El cosechero tercerizado cosecha entre un 70 o $60 \%$ más volumen de fruta que el cosechero de la casa. La figura del patrón está más arriba de ellos y eso hace que el cosechero rinda más." (Gerente RRHH empresa 1). Añaden que, aunque paguen más por la tercerización, les rinde más la cosecha. Finalmente aseguran que es una ventaja para los y las trabajadoras, ya que "El cosechero es un tipo que vive el día a día. Le pide al patrón un adelanto. Tiene contacto directo con él. Eso es una ventaja para el cosechero". (Gerente RRHH empresa 1, abril de 2018).

Encontramos así una serie de argumentos de peso para explicar las motivaciones de la empresa a tercerizar, que se vinculan siempre a la efectividad del contratista. Esta capacidad radica en el conocimiento directo de los y las trabajadoras, así como en un vasto manejo de las dinámicas locales, aspectos que lo colocan en un lugar estratégico para la mediación en las relaciones laborales. A lo largo del trabajo de campo se reitera, tanto en palabras de contratistas como cosecheros y cosecheras, esta cualidad del contratista para estar cerca de las necesidades de la gente: es frecuente que los contratistas realicen préstamos o adelantos de dinero informales e incluso encontramos casos donde los contratistas otorgaban préstamos para la compra de vehículos livianos o electrodomésticos en planes de pago accesibles para los y las trabajadoras, comprometiendo trabajo en la zafra siguiente.

Otra dimensión importante del fenómeno son las articulaciones políticas que muchos contratistas mantienen a nivel de gobierno local, facilitando la resolución de necesidades comunitarias, sea o bien porque ellos mismos son a su vez funcionarios jerárquicos de los gobiernos locales o porque mantienen fuertes lazos con figuras de peso. También es frecuente que, por la vía de otros negocios, ya sea de su entera pertenencia o de vínculos cercanos, realicen donaciones para facilitar el funcionamiento de las organizaciones sociales locales, como ser los clubes de baby fútbol, las comisiones de fomentos de las escuelas, etc. De esta manera, cuando las relaciones marchan favorablemente, el contratista es una suerte de prohombre, o patriarca dadivoso. El conocimiento denso del territorio y sus dinámicas, la cercanía con las personas, su capacidad de erogación económica y de mediación política, se configuran como habilidades blandas (Heckman y Katz, 2012) para la gestión efectiva de la mano de obra a nivel local, a la vez que para la viabilización de los intereses de las empresas.

En contraposición, los informantes de otras tres empresas entrevistadas señalaron menos convicciones respecto a las bondades de la tercerización. Uno de ellos dijo "contratar algo de la mano de obra a través de empresas tercerizadas, pero muy poco" (gerente de planta de exportación empresa 2, agosto de 2019), mientras las otras dos empresas citrícolas no usan este mecanismo de contratación de mano de obra. Incluso 
en un caso, la empresa hizo una prueba piloto de tercerizar el manejo de la mano de obra, con resultados desalentadores.

\begin{abstract}
Hubo una experiencia en el año 2015-2016 de tercerizar algo de cosecha en el sur y la verdad que yo la prohibí al año. Porque ¿qué es lo que pasa cuando vos tercerizás? Cuando vos tercerizás trabajo de campo o de cosecha, si a vos como empresa te sale más barato, es porque algo el contratista no está pagando. Porque a vos te tiene que cobrar el servicio, una ganancia tiene el contratista, entonces ¿̨cuánto te llega a vos que sos empleado del contratista? Cuando te tiene que aplicar el mismo convenio que aplica para el citrus. Significa que o no te aplica el mismo convenio o no aporta todo lo que tiene que aportar para la seguridad social. Entonces esa es la cuenta, si a vos tercerizar te sale más barato que ser empleado tuyo hay algo que el tercero se está llevando (...) ¿quién sufre en esa cadena? el trabajador y el Estado. Porque a donde va a ir más fácil es a declarar la cantidad mínima de jornales para que tenga cobertura médica que nos salve y la otra cantidad de jornales no la declaro y pago menos aportes. Ahí es donde está la ganancia del contratista (...).

E: ¿Y cuál es el atractivo de tercerizar?

Empresa 4: Y abaratás el costo de la cosecha, el incentivo es abaratar, pero a costa del trabajador y tarde o temprano te va a venir a vos un reclamo. Por eso es que fue esa prueba piloto y dijimos: no va más. (Gerente de RRHH empresa 4 , comunicación personal, agosto de 2019).
\end{abstract}

Así, el mecanismo de tercerización de la mano de obra implica siempre una negociación constante y continua entre los diferentes actores (empresa, trabajadores) pero también con las normas, ya que son las empresas contratistas quienes generan deudas con el Banco de Previsión Social (BPS) y las que en ocasiones pagan de su bolsillo hasta tanto entren los pagos:

\footnotetext{
Yo tengo que ir a conveniar ahora con el BPS los pagos atrasados de los cuatrimestres. Porque las empresas tercerizadas, la nuestra, o sea todas jestamos todos atrasados! todos haciendo convenio con el BPS, ¿no? aparte de tener el embargo, de tener la denuncia judicial y la denuncia penal, por los montos que somos... y bueno ahí andamos, tampoco te sirve ir a pelearte con la citrícola por qué no ganas nada. A mí lo que me interesa es que me empiecen a dar los cheques. (Contratista 1, comunicación personal, abril de 2018).

Hace 5, 6 años que este rubro tiene temas económicos bastante complicados... tenés que estar llorando por un cheque, peleándose. (...) En mi rubro vos tenés que trabajar con un colchón de plata, no te digo un colchón lleno, tenés que tener cierto respaldo. Que yo sepa que yo llego al viernes pagando lo que trabajé. Después tengo que hacer mi trabajo: cobrárselo a los productores, ta? (...) si te dan, te dan un cheque diferido, el cheque diferido tenés que depositarlo y esperar que te llegue la fecha. No está fácil. (Contratista 2, comunicación personal, abril de 2018).
}

\title{
5.2 Los sentidos asociados a la feminización de la mano de obra
}

Respecto a la feminización de la mano de obra, tanto los contratistas como los gerentes de recursos humanos señalan que se trata de un cambio reciente y las estimaciones sugieren una participación de asalariadas mujeres en la cosecha del citrus que ronda entre el 20 y el $40 \%$ del total de la mano de obra: "Antes, cuando yo arranqué, si no eras hombre no te llevaban a trabajar. (...) 30 años atrás. (...) con el tiempo fue creciendo y creciendo la mano de obra femenina. Y hoy yo no sé si no está en el $40 \%$ ya" (contratista 3, febrero de 2018).

Al indagar sobre las causas, se marca como hito el cambio de la 'camisa' al 'bolso', que alivianó el peso a cargar. ${ }^{4}$ A su vez, si atendemos al período temporal en que se da la feminización, coincide con años de reactivación económica (boom de la construcción y de la soja) que generaron alternativas para la mano de obra masculina. A su vez, tanto los contratistas como los gerentes de recursos humanos, ponderaron favorablemente el cambio hacia una mayor participación femenina en la cosecha de los cítricos. La característica con mayor frecuencia señalada fue la presencialidad. Además, se señala el trabajo prolijo y mejor disciplina en general. Uno de los contratistas incluso señala que "las mujeres trabajan mucho más que los hombres" (contratista 4) y desde recursos humanos de una de las empresas se plantea que las mujeres son mejores para desempeñar tareas de sanidad. 
En las mujeres tenés más asistencia, tenés trabajo más prolijo. Nosotros decimos siempre que la mujer te mejora el grupo, o sea el tipo ya no grita tanto, ya no se va tanto de boca, las bromas son distintas, o sea, si la mujer marca el nivel, el grupo está mucho mejor seguro, se cuidan más, hasta el ómnibus más prolijo. (contratista 2, comunicación personal, abril de 2018).

Otro contratista, además de la importancia del presentismo, menciona a la mujer trabajadora como señuelo para lograr la asistencia del trabajador varón:

Lo que te hace es la constancia de que si vos vas a tener un hombre que va 4 días por semana y te cosecha 50 cajones y te va 4 , te hace 200 y la mujer te va los 6 y te hace 35 , te sigue sirviendo la mujer, que te hace 210 cajones a la semana. (...) Yo decía que era mejor tener mujeres porque me llevaba a los hombres, porque si había 10 mujeres, siempre alguno se quería hacer el lindo e iba. (contratista 3, comunicación personal, febrero de 2018).

Por otro lado, aparece en los discursos la doble jornada laboral de las mujeres como un elemento que funciona como una desventaja para su empleabilidad.

¿Cuándo vos perdés personal femenino? la que tiene que llevar el hijo a la escuela, la que tiene que estar a la hora que lo tiene que ir a buscar, ahí vas perdiendo. El hombre como que no, como que está más disponible para todas esas cosas, podés hacerte un viaje lejos. Cuando te toca una chacra a $80 / 90 \mathrm{~km}$ ya perdés personal femenino. De vuelta, llegamos muy tarde. Y ni siquiera es porque le pagues una hora más, la tipa no llega a tiempo para las cosas de su casa (contratista 2, comunicación personal, abril de 2018).

Además, los contratistas reconocen lo pesado de la tarea de cosechar y cómo esto puede hacerse sentir en el rendimiento de las mujeres.

De repente la mujer le cuesta un poco más (...) la escalera se torna pesada, el bolso es pesado, si vos tenés toda la logística pronta como para trabajar cerca de las plantas, los bins, lugar de acopio donde volcás la fruta y que no tengas que caminar tanto con el bolso de cosecha, no se notan las diferencias, ahora, donde estén un poco judiada la cosa, si se nota (contratista 2, comunicación personal, abril de 2018).

Finalmente, los embarazos se mencionan como una realidad inevitable: “y yo tengo 1 o 2 por zafra (...) "que va hace" (...) Y bueno no te queda otra. Vos no le podés preguntar si se va a embarazar o no..." (Contratista 3, comunicación personal, febrero de 2018). Las mujeres embarazadas requieren realizar gestiones en el BPS por el contratista que demandan cierta cantidad de tiempo y que impiden a la empresa "cerrar la caja" cuando termina la cosecha, hasta tanto la trabajadora no termine el período de gestación.

\section{Conclusiones}

En este trabajo mostramos cómo en el Uruguay los cambios acaecidos en un sector claramente inserto en una CGV, como lo es el sector de la citricultura, han implicado transformaciones en la organización del trabajo, las formas de contratación del personal y la participación de las mujeres. En ese marco, nos propusimos analizar el incremento de la mano de obra femenina en la fase agraria del rubro citrícola, haciendo hincapié en los procesos de jornalerización y buscando al mismo tiempo dar cuenta de los sentidos otorgados por algunos actores empresariales a esos procesos.

En cuanto a la creciente tendencia a la feminización y jornalerización del trabajo asalariado en el sector, los datos analizados nos permitieron constatar un incremento en la participación de fuerza de trabajo femenina, así como una tendencia creciente hacia contratos temporales (jornalerización). En concreto, mientras el número de asalariados varones se incrementó $31 \%$ entre 1990 y 2011, el número de asalariadas mujeres aumentó $382 \%$, es decir, doce veces más.

Por otra parte, si bien la fruticultura en 1990 y en 2000 dependía mayoritariamente de trabajadores/as permanentes, hacia 2011 pasó a utilizar principalmente mano de obra jornalera. En esta misma línea, los datos del 2011 nos permiten confirmar que la realidad de la citricultura profundiza la tendencia que se observa en general para la fruticultura. En el rubro citrícola, la participación de las mujeres en el trabajo se asocia con 
un tipo de vínculo contractual: el jornalero. De hecho, el peso de las mujeres asalariadas temporales de la citricultura triplica el de las permanentes.

Luego, a través de un enfoque cualitativo, buscamos dar cuenta de las motivaciones y razones que actores de las principales empresas citrícolas del país, así como contratistas, encuentran y construyen en relación a estos procesos. En ese camino constatamos que el proceso de tercerización de la mano de obra forma parte de un proceso de reorganización del trabajo comandado y dirigido desde las empresas madres. Y este proceso fue concurrente al proceso de feminización de la mano de obra, así como a su creciente jornalerización.

En síntesis, consideramos que nuestro trabajo realiza un aporte al conocimiento acerca de los procesos y mecanismos que se utilizan en las CGV para controlar la mano de obra, en el marco de la tendencia más general a bajar los costos de la misma (Hopkins y Wallerstein, 1994). En ese sentido, presentamos evidencia en relación a cómo la tercerización, la jornalerización y la feminización, se complementan y potencian generando un efecto sinérgico, funcional a los intereses del capital, expresados en las estrategias de las principales empresas del rubro.

Mostramos cómo la tercerización y la jornalerización, aun cuando en el papel podrían implicar mayores costos, en la práctica habilitan una serie de mecanismos de control sobre la mano de obra -corporizados en la figura del contratista- así como una serie de espacios que escapan a la regulación -subdeclaración de jornales y aportes al Estado- que alivianan los costos para la empresa y mejoran su rentabilidad. De este modo, la proliferación de las empresas contratistas de mano de obra permite prácticas (como la subdeclaración o el subregistro) que a las grandes empresas les resultan impracticables por su propio peso y notoriedad.

Al mismo tiempo, la cercanía de los contratistas con la mano de obra habilita también el manejo y la creación de un conjunto de lazos de dependencia que permiten sostener -muchas veces mediante intercambios materiales y simbólicos externos al vínculo laboral- unas condiciones de trabajo precarias.

Por otro lado, mostramos también cómo la feminización potencia los mecanismos anteriores. Los análisis cuantitativos realizados evidencian la concurrencia temporal de los fenómenos de jornalerización y feminización, al tiempo que las razones y motivos esgrimidos por los actores empresariales nos mostraron la "contribución" que la desigualdad de género realiza, como condición de posibilidad para los otros procesos. Así, de acuerdo al discurso empresarial, el presentismo, la responsabilidad y la constancia son los factores que transforman a las mujeres en un activo atractivo. La feminización permite hacer frente al problema central del contratista: encontrar mano de obra disponible, confiable y controlable, dispuesta a aceptar las peculiares condiciones de trabajo.

Estos hallazgos empíricos cobran nuevos sentidos desde la lectura feminista que proponemos. El concepto de "circuito amplio de trabajo" (Carrasco, 2014) permite visibilizar que el trabajo productivo, generalmente asalariado, se sostiene en forma integrada y dinámica sobre el trabajo reproductivo, es decir las tareas domésticas y de cuidados. Sabemos que el trabajo reproductivo se distribuye socialmente en forma desigual y recae mayoritariamente sobre las mujeres. Las mujeres ingresan al trabajo productivo (asalariado) pero no renuncian al trabajo reproductivo (doméstico) y, a su vez, en muchos casos, enfrentan solas el cuidado de los hijos, hijas y familiares a cargo. Como mecanismo conexo, proponemos atender a la dinámica que adquiere la deuda como factor regulador de las relaciones laborales y particularmente a los procesos de "feminización de la deuda". El salario no alcanza para cubrir los costos reproductivos, por lo cual trabajadoras y trabajadores se ven forzados a endeudarse, comprometiendo sus posibilidades de acción a futuro (Cavallero y Gago, 2019). Nos referimos a un tipo particular de deuda que se adquiere para necesidades básicas del núcleo familiar, como alimentación, vestimenta, equipamiento básico del hogar y útiles escolares, entre otras cosas. Dada la desigualdad estructural en las responsabilidades de cuidados, este tipo de deuda recae mayormente sobre las mujeres. Las asalariadas rurales endeudadas pierden autonomía a la hora de negociar remuneraciones y condiciones laborales precarias, mecanismo que muchas veces se ve agravado en algunas situaciones puntuales que encontramos en el trabajo de campo, en donde los contratistas adelantan dinero, o dan facilidades de crédito en negocios propios, a las trabajadoras. 
Así, los hallazgos analizados a la luz de la discusión conceptual presentada nos permiten sostener que existe una complicidad estructural entre las desigualdades de género, producidas por el sistema patriarcal, y las necesidades de acumulación mediante explotación del trabajo del sistema capitalista.

\section{BibliografíA}

Ackermann, M. y Díaz, A. (2018). Fruticultura: situación y perspectivas de la citricultura y los frutales de hoja caduca. En Ministerio de Ganadería, Agricultura y Pesca (2018) Anuario 2018 OPYPA. Análisis sectorial y cadenas productivas. Temas de politica. Estudios (pp. 253-277). Disponible en: http://www.mgap.gub.uy/sites/default /files/15 anuario 2018 - fruticultura.pdf

Aranda, X. (1988). Elnuevo protagonismo social femenino y sus escenarios en los años ochenta. Santiago de Chile: CEPAL. Recuperado de https://repositorio.cepal.org/bitstream/handle/11362/16818/S8881102 es.pdf

Bamber, P. y Staritz, C. (2016). The gender dimensions of Global Value Chains. Geneva: International Centre for Trade and Sustainable Development (ICTSD). Recuperado de https://www.ictsd.org/sites/default/files/research/the gender dimensions of global value chains 0.pdf

Basualdo, V. y Esponda, M. (2014). La expansión de la tercerización a nivel global a mediados de los años setenta, sus antecedentes históricos y su alcance actual. En V. Basualdo y D. Morales (coords.), La tercerización laboral: Origenes, impacto y claves para su análisis en América Latina (pp. 19-64). Buenos Aires: Siglo XXI.

Bendini, M. y Pescio, C. (1996). Trabajo y cambio técnico. El caso de la agroindustria frutícola del Alto Valle. Buenos Aires: La Colmena.

Bericat, E. (1998). La integración de los métodos cuantitativo y cualitativo en la investigación social. Barcelona: Ariel.

Carámbula, M. (2015). Imágenes del campo uruguayo en clave de metamorfosis. Cuando las bases estructurales se terminan quebrando. Revista de Ciencias Sociales 28(36), 17-36. Recuperado de http://cienciassociales.edu.uy/departamentodesociologia/wp-content/uploads/sites/3/2015/09/Art\%C3 \%ADculo-Car\%C3\%A1mbula-RCCSS-36.pdf

Carámbula, M. y Piñeiro. D. (2010). Ciclo anual de trabajo y precariedad laboral subjetiva de los esquiladores de Villa Sara. Agrociencia Uruguay 14(1). Recuperado de http://www.scielo.edu.uy/scielo.php?script=sci_arttextypid= $\underline{\text { S2301-15482010000100009 }}$

Carámbula, M., Figueredo, S. y Bianco, M. (2013). Resolviendo las necesidades del capital. Del intercambio laboral a la empresa de servicios agrícolas. Revista de Ciencias Sociales 26(32), 35-52. Recuperado de http://cienciassociales.edu.uy/departamentodesociologia/wp-content/uploads/sites/3/2013/archivos/Rev ista\%20de\%20Ciencias\%20Sociales\%2032.pdf

Cardeillac, J. (2013). Documento de Trabajo No 88: Sobre Cadenas Globales de Valor. Montevideo: NESA -DS-FCS. Recuperado de https://www.colibri.udelar.edu.uy/jspui/bitstream/20.500.12008/4595/1/DT\%20S\%202013 \%2088.pdf

Cardeillac, J. (2019). Las Transformaciones del agro uruguayo entre 1990 y 2011 desde una perspectiva de la Estructura Agraria: descomposición de la producción familiar, acaparamiento de tierras por centralización de capital y polarización. (Tesis de doctorado en Sociología). Facultad de Ciencias Sociales, UDELAR.

Cardeillac, J. y Nathan, M. (2015). Caracterización socio-demográfica de la situación de los colectivos de trabajadores rurales y domésticos en el período 1996-2011. En: F. Pucci, D. Piñeiro, A. Juncal y S. Nión (coord.), Sindicalización y negociación en los sectores rural y doméstico (pp. 55-81). Montevideo: Mundo Gráfico SRL.

Cardeillac, J. y Piñeiro, D. (2017). Cambios en la producción familiar y empresarial del Uruguay entre 2000 y 2011. El debate entre Lenin y Chayanov revisitado. Revista Latinoamericana de Estudios Rurales (ALASRU) 2(4), 109-138. Recuperado de http://www.ceil-conicet.gov.ar/ojs/index.php/revistaalasru/article/view/323

Cardeillac, J. y Rodríguez Lezica, L. (2018). Exclusión en la inclusión por descalificación: análisis de la situación de las asalariadas rurales en Uruguay. Revista Nera 21(41), 138-164. Recuperado de http://revista.fct.unesp.br/inde x.php/nera/article/view/5387 
Cardeillac, J., Mascheroni, P. y Vitelli, R. (2016). Investigación sobre definición operativa de la población "rural” con fines estadísticos en Uruguay. MIDES.

Cardeillac, J.; Gallo, A. y Juncal, A. (2015). Permanencias en el agro uruguayo: Un estudio de caso sobre el trabajo asalariado rural. Revista de Ciencias Sociales, 28(36), 77-98. Recuperado de http://cienciassociales.edu.uy/departamentodesociologia/wp-content/uploads/sites/3/2015/09/Art\%C3 \%ADculo-Cardeillac-Gallo-Juncal-RCCSS-36.pdf

Carrasco, C. (2014). La economía feminista: ruptura teórica y propuesta política. En C. Carrasco (comp.), Con voz propia (pp. 25-47). Madrid: La oveja roja.

Cavallero, L. y Gago, V. (2019). Una lectura feminista de la deuda. Vivas, libres y desendeudadas nos queremos. Buenos Aires: Fundación Rosa Luxemburgo.

Chiappe, M., Carámbula, M. y Fernández, E. (2008). El campo uruguayo: una mirada desde la sociología rural. Montevideo: Departamento de Publicaciones de Facultad de Agronomía. Universidad de la República.

Creswell, J. W. (2014). Research design: qualitative, quantitative, and mixed methods approaches. California: SAGE Publications, Inc.

Dalla Costa, M. R. (1972). Las mujeres y la subversión de la comunidad. México: Siglo XXI.

Dirección de Estadísticas Agropecuarias. (2018). Encuesta Citrícola "Primavera 2018". 26 de noviembre de 2018. Recuperado de http://www.mgap.gub.uy/sites/default/files/publicacion_primavera_2018.pdf

Dominzain, S. (2003). Las trabajadoras del cítrico en Uruguay. Condiciones de trabajo y derechos laborales. Revista Estudios del Hombre, 16, 243-267. Disponible en: http://148.202.18.157/sitios/publicacionesite/pperiod/est hom/esthompdf/esthom16/9.pdf

Ermida Uriarte, O. y Orsatti, A. (2009). Estrategia sindical hacia trabajadores tercerizados. GTAS, Confederación Sindical de Trabajadores de las Américas.

Errea, E., Peyrou, J., Secco, J. y Souto, G. (2011). Transformaciones en el agro uruguayo: Nuevas instituciones y modelos de organización empresarial. Montevideo: Universidad Católica del Uruguay.

FAO, FIDA y OIT (2010). Politicas de mercado de trabajo y pobreza rural en América Latina, FAO.

Federici, S. (2013). Revolución en punto cero. Trabajo doméstico, reproducción y luchas feministas. Madrid: Traficantes de sueños.

Gabinete Productivo (2010). Cadenas de valor (II): Bio y Nanotecnología, avicola, porcina, citricos, textil, vestimenta. Montevideo: Mastergraf. Disponible en: http://www.agev.opp.gub.uy/observatorio docs/publico/197.pdf

Galcerán Huguet, M. (2006). Introducción: Producción y reproducción en Marx. En Transformaciones del trabajo desde una perspectiva feminista. Producción, reproducción, deseo, consumo. Madrid: Tierradenadie Ediciones.

Galván, M. (18 de julio de 2013). No contaban con mi astucia. La Diaria. Recuperado de: https://ladiaria.com.uy/ar ticulo/2013/7/no-contaban-con-mi-astucia/

Gereffi, G., Korzeniewicz, M. y Korzeniewicz, R. P. (1994). Introduction: Global Commodity Chains. En G. Gereffi y M. Korzeniewicz, Commodity chains and global capitalism. USA: PraegerPublishers.

Heckman, J. y Katz, T. (2012). Hard evidence on soft skills. National Bureau of Economic Research. Working Paper Series $N^{\circ}$ 18121. Disponible en: https://www.nber.org/papers/w18121.pdf

Hernández López, J. (2014). La jornalerización en el paisaje agavero. Actividades simples, organización compleja. Ciudad de México: CIESAS.

Hopkins, T. K. y Wallerstein, I. (1994). Commodity Chains in the Capitalist World-Economy Prior to 1800. En G. K. Gereffi y M. Korzeniewicz, Commodity chains and global capitalism. USA: Praeger Publishers. http://148.2 02.18.157/sitios/publicacionesite/pperiod/esthom/esthompdf/esthom16/9.pdf

Ipsen, I. (2010). Ni Peras ni manzanas: la transición de género en los puestos de trabajo en los empaques de citricos en Uruguay. Ponencia presentada al VIII Congreso Latinoamericano de Sociología Rural, Porto de Galinhas, Brasil.

Juncal, A., Cardeillac, J., Moreira, B., y Gallo, A. (2014). Conceptualización de asalariados agropecuarios y caracterización de sus condiciones de vida en un contexto de crecimiento económico y desarrollo social del Uruguay. En M. Boado (coord.), El Uruguay desde la Sociología 12 (pp. 259-271). Montevideo: Mastergraf S.R.L. 
Recuperado de http://cienciassociales.edu.uy/departamentodesociologia/wp-content/uploads/sites/3/2014/0 6/El-Uruguay-desde-la-Sociologu00EDa-XII.pdf

Lara Flores, S. (1991). Las obreras agrícolas: un sujeto social en movimiento. Nueva Antropología, XXI(39), 99-114. Recuperado de http://www.redalyc.org/articulo.oa?id=15903907

Lastarria-Cornhiel, S. (2008). Feminización de la agricultura en América Latina y África. Tendencias y fuerzas impulsoras. Chile: RIMISP.

Mingo, E. (2015). Resistentes, comprometidas y conflictivas: obreras de la agroindustria frutícola en Argentina. Una mirada desde la demanda de mano de obra. En A. Riella y P. Mascheroni (Ed.), Asalariados rurales en América Latina (pp. 289-312). Montevideo: Doble clic Editoras.

Neiman, G. (2010). Los estudios sobre el trabajo agrario en la última década: una revisión para el caso argentino. Mundo Agrario, 10(20). Recuperado de https://www.mundoagrario.unlp.edu.ar/article/view/v10n20a20/479

OPyPA - MGAP. (2017). Anuario OPyPA 2017. Montevideo: MGAP - OPyPA. Disponible en: http://www.mgap.gub.uy/unidad-organizativa/oficina-de-programacion-y-politicas-agropecuarias/publica ciones/anuarios-opypa/2017

OPyPA - MGAP. (2018). Anuario OPyPA 2018. Montevideo: MGAP - OPyPA. Disponible en: http://www.mgap.gub.uy/unidad-organizativa/oficina-de-programacion-y-politicas-agropecuarias/publica ciones/anuarios-opypa/2018

Piñeiro, D. (2010). Concentración y extranjerización de la tierra en el Uruguay. En M. Manzanal y G. Neiman (coords.), Las agriculturas familiares del MERCOSUR. Trayectorias, amenazas y desafios (pp. 153-170). Buenos Aires: CICCUS.

Piñeiro, D. (2011). Precariedad objetiva y subjetiva en el trabajo rural: nuevas evidencias. Revista de Ciencias Sociales, 24(28), 11-33. Recuperado de https://hdl.handle.net/20.500.12008/6880

Piñeiro, D. (2014). Asalto a la tierra: el capital financiero descubre el campo uruguayo. En G. Almeyra, L. Concheiro Bórquez, J. M. Mendes Pereira y C. W. Porto-Gonçalves (comp.), Capitalismo: tierra y poder en América Latina (1982-2012). Argentina, Brasil, Chile, Paraguay, Uruguay. Volúmen I. Buenos Aires: Ediciones Continente.

Quaranta, G. y Fabio, F. (2011). Intermediación laboral y mercados de trabajo en agriculturas reestructuradas: el caso del Valle de Uco, Mendoza, Argentina. Región y Sociedad, 23(51), 193-225. Recuperado de http://www.scielo .org.mx/scielo.php?script $=$ sci arttextypid $=$ S1870-39252011000200007

Riella, A. y Mascheroni, P. (2016). Intermediación laboral en los mercados de empleos rurales en Uruguay. Eutopia, 9, 29-43. Recuperado de https://doi.org/10.17141/eutopia.9.2016.2062.

Riella, A., y Romero, J. (2014). Continuidades y rupturas en la estructura agraria en el Uruguay del siglo XXI. PAMPA, 1 (10), 159-171. Disponible en: https://bibliotecavirtual.unl.edu.ar/publicaciones/index.php/PAMPA/articl e/view/4535

Rodríguez Lezica, L. (2014). Entre la inclusión y el olvido. La cuestión de género en el trabajo asalariado rural: el caso de la citricultura uruguaya (Tesis de Maestría). Quito: FLACSO Ecuador.

Rodríguez Lezica, L. (2018). ¿Y las mujeres dónde están? Una otra mirada al sindicalismo rural en Uruguay. Revista Interdisciplinaria de Estudios Agrarios, 49, 5-34.

Rodríguez Lezica, L. y Carámbula, M. (2015). Las olvidadas de la tierra: asalariadas rurales del Uruguay. Clase y género en cuestión. Revista Agrociencia, 19(2). Recuperado de http://www.fagro.edu.uy/agrociencia/index.php/direc torio/article/view/1154

Ruffier, J. (2005). La frontera como ventaja competitiva: la producción de naranjas en ambas márgenes del río Uruguay. Revista Galega de Economia, 14(1-2). Recuperado de http://www.redalyc.org/articulo.oa?id=39114221

Salamea, L. y Waters, W. (1995). La cuestión de género en la reestructuración de la agricultura ecuatoriana. En S. Lara Flores (coord.), Jornaleras temporeras y bóias-frias: El Rostro Femenino del Mercado de Trabajo Rural en América Latina. Caracas: Nueva Sociedad.

Soto Baquero, F. y Klein, E. (coord.) (2012). Políticas de mercado de trabajo y pobreza rural en América Latina. Tomo II. Roma, Italia: FAO. 
Sturgeon, T. J. (2008). From Commodity Chains to Value Chains: Interdisciplinary theory building in an age of globalization. Massachusetts: MIT-IPC-08-001.

Tadeo, N., Palacios, P. y Torres, F. (2005). Zafralidad y empleo en la citricultura entrerriana. Revista Interdisciplinaria de Estudios Agrarios, 22, 5-30. Recuperado de http://www.memoria.fahce.unlp.edu.ar/art revistas/pr.11139/ pr.11139.pdf

Todaro, R. y Yáñez, S. (2004). El trabajo se transforma. Relaciones de producción y relaciones de género. Santiago, Chile: Centro de Estudios de la Mujer.

\section{ENTREVISTAS}

Contratista 1: Entrevista realizada en abril de 2018, en Villa Constitución (departamento de Salto)

Contratista 2: Entrevista realizada en abril de 2018 en la ciudad de Salto.

Contratista 3 y contratista 4: Entrevista grupal realizada en febrero de 2018 en la ciudad de Salto.

Gerente de recursos humanos de empresa 1: Entrevista realizada en abril de 2018 en la ciudad de Salto.

Gerente de planta de exportación de empresa 2: Entrevista realizada en agosto de 2019 en la ciudad de Salto.

Gerente de recursos humanos de empresa 3: Entrevista realizada en agosto de 2019 en la ciudad de Paysandú.

Gerente de recursos humanos de empresa 4: Entrevista realizada en agosto de 2019 en la ciudad de Young.

\section{Notas}

1 Si bien se podría plantear que la tercerización del trabajo es sólo una de las funciones del intermediario o la empresa de servicios -algo que se identifica con claridad en otros rubros, como la soja y la forestación, en donde la intermediación no sólo se explica en la dimensión laboral, sino también financiera, tecnología, logística, cultural (Carámbula, Figueredo y Bianco, 2013)-, en el caso del citrus, la tercerización del trabajo y los trabajadores aún mantiene formas tradicionales de intermediación (Riella y Mascheroni, 2016).

2 Dentro de la cadena pueden reconocerse 2 grandes fases. La fase agraria es la que se dedica a la producción de la fruta, los plantines en los viveros, la plantación y la cosecha. La fase industrial es aquella en la que se realiza el empaque de la fruta para exportar, así como el procesamiento de jugos concentrados, aceites esenciales y pellets.

3 Los censos de población permitirían la desagregación, pero no relevan las variables ocupacionales y las Encuestas Continuas de Hogares no permiten la desagregación necesaria.

4 La antigua camisa naranjera cargaba hasta 70 kilos de naranjas. Actualmente se usa un bolso que carga como máximo 12 kilos de naranjas.

* El presente trabajo se enmarca en el proyecto de investigación y desarrollo "Procesos de trabajo y desigualdades de género en dos cadenas de valor agroindustriales: citrus y lechería", financiado por la Comisión Sectorial de Investigación Científica (SCIC). 